\title{
The delivery of Emergency Medicine at the Teaching Hospital Karapitiya Emergency Treatment Unit - An Australian Perspective
}

\author{
Davis $\mathbf{A}^{1}$, Taylor $\mathbf{N}^{2}$, O'Reilly $\mathbf{G} \mathbf{M}^{3,4,5}$ Vidanapathirana $\mathbf{T}^{6}$, Uluwattage $\mathbf{W} \mathbf{H}^{6}$ \\ Journal of the Ceylon College of Physicians, 2017, 48, 20-23
}

\section{Scenario 1: A patient of the THKETU}

A 14-year-old boy is transferred to the Teaching Hospital Karapitiya Emergency Treatment Unit (THK ETU) with fever and collapse. He is immediately triaged to a resuscitation bay and met by a team of highly skilled nursing and medical staff. He is found to be hypotensive, hypoxic and profoundly acidotic. A bedside ultrasound reveals bilateral pleural effusions and a large volume of free intraperitoneal fluid. He is managed as dengue haemorrhagic shock and within one hour multiple interventions have taken place. In this time, he is intubated, ventilated (to ARDS-NET principles) and has a central line. Hyperkalaemia has also been reversed, a blood transfusion and pressors have been commenced and intra-abdominal compartment pressures have been estimated. A paediatrician and renal physician have reviewed him and he is ready for transfer to an intensive care bed.

Emergency Medicine (EM) is a relatively new specialty to Sri Lanka and there is a strong vision and investment in its expansion and success. EM is the field of practice that provides care to undifferentiated patients with acute injury or illness where timely intervention is required to reduce morbidity and mortality. This case demonstrates how a team of trained staff, within the framework of an Emergency Treatment Unit, can rapidly

\footnotetext{
1 Emergency Department, Geelong Hospital, Geelong, Australia.

${ }^{2}$ Emergency Department, Canberra Hospital, Canberra, Australia.

${ }^{3}$ Emergency and Trauma Centre, The Alfred Hospital, Melbourne, Australia.

${ }^{4}$ National Trauma Research Centre, The Alfred Hospital, Melbourne, Australia.

${ }^{5}$ School of Public Health and Preventive Medicine, Monash University, Melbourne, Australia.

${ }^{6}$ Teaching Hospital Karapitiya, Galle, Sri Lanka.
}

Corresponding author: Davis A

E-mail: annalouisedavis@gmail.com and skillfully deliver complex critical care and simultaneously gather specialist resources.

The Teaching Hospital Karapitiya is the main tertiary care provider in the South and boasts 1560 beds, 54 wards, 500 admissions / day, a full suite of specialties and an affiliation with the co-located University of Ruhuna. The THK ETU was developed as part of a Sri LankanAustralian partnership as a component of the response to the devastating tsunami of 2004, and is now one of the most established ETUs in Sri Lanka. THK ETU is a bustling department with 4 resuscitation, 12 acute care and 8 short stay beds. Its staffing model consists of three general physicians with an interest in emergency medicine, 1 paediatrician, 6 emergency registrars, 23 residents and 45 nursing staff. It provides a 24 hour service, 7 days a week and has a strong commitment to training and EM development.

\begin{abstract}
Aims
The aim of this narrative is to illustrate important aspects of the THK ETU model of care and the role that EM plays within the hospital and the local health system. These factors are highlighted in several cases and may serve to clarify the scope of practice of EM as a specialty. A further aim of this piece is to describe the vision that KTH ETU has for the future, and so facilitate a collaborative approach to the development of ETUs across Sri Lanka.
\end{abstract}

\section{Scenario 2: The role of triage}

A 50-year-old lady, ambulant at triage, presents to THK ETU with acutely worsening lethargy and faintishness. The triage nurse notes a blood pressure of 90/50 and allocates a triage category of one, necessitating immediate medical review. She is transferred to a resuscitation cubicle where the emergency resident notes features of profound hypothyroidism and a large pericardial effusion with early features of tamponade on bedside echocardiogram. Despite initial treatment, her blood pressure deteriorates to $75 / 40$, prompting a rapid referral to the on call cardiologist. A short time following her presentation to the THK ETU the cardiologist inserts a pericardial and the patient's haemodynamics normalize. 
A major challenge for emergency departments worldwide is to efficiently identify truly emergent patients whilst safely diverting more stable cases to other suitable care. Good triage practices are indispensable to a safe hospital. This case highlights how triaging rapidly to the most appropriate clinical area led to a time critical intervention being performed.

The nurses of THK ETU show great expertise in the application of emergency triage to some 300 daily presentations. The patients' clinical urgency is determined by a combination of presenting complaint and vital signs. Details are documented on a proforma and the patient is allocated to a four-point triage scale, adapted for local use from the Australasian 5-point triage score.

All patients with an immediate or imminent threat to life or limb are cared for in the ETU. Patients with acute severe sepsis, major trauma, status epilepticus, significantly deranged vital signs and severe pain are frequent examples. A narrow-spectrum of more stable patients who will benefit from urgent care are also cared for in the ETU. These patients are often more difficult to identify and rely on an astute triage nurse bolstered by education, guidelines and medical support. Examples include the seemingly well patient with febrile neutropaenia, the 'leaking' dengue patient, the septic neonate, many patients with chest pain and those with more subtly deranged vital signs.

Patients deemed less urgent are reviewed briefly for 2 to 3 minutes by an "admission resident" to further determine their suitability for the ward. These patients are then sent directly to an inpatient bed for review.

\section{Scenario 3: Team based care}

A 32-year-old motorbike rider with a life-threatening combination of injuries is brought to THK ETU via threewheeler. He is immediately triaged to the resuscitation bay where he is met by a 'trauma team' consisting of 3 residents and 4 nurses. The patient is hypotensive, tachycardic and hypoxic. He has Le Forte III facial fractures threatening his airway, multiple rib fractures, a right-sided tension pneumothorax and intra-abdominal bleeding. An 'airway doctor and nurse' rapidly institute spinal precautions, intubate the patient and pack his nasal and oral cavity with ribbon gauze. An 'assessment doctor' performs a primary survey consisting of a clinical examination, a brief history, a bedside ultrasound and a 'log roll' to promptly identify immediate life-threats. A 'monitoring nurse' swiftly undresses the patient, attaches him to the monitor and begins preparing necessary resuscitation medications and fluids. A 'procedure nurse' and 'procedure doctor'gain wide-bore bilateral IV access, insert an intercostal catheter and activate a massive transfusion protocol. A designated 'team leader' coordinates care and communicates with the haemotolgist, general surgeons, ear nose and throat surgeons and anaesthetists. The patient is safely delivered to the operating theatre within a short time of arrival.

Integral to emergency medicine is the team-based resuscitation of critically ill patients. Groups of nurses and doctors with pre-determined and practiced roles may identify and relieve multiple life threats in parallel. This is performed with an efficiency that may be out of reach of the sole practitioner. This case also demonstrates the role of the emergency physician and the ETU as a focal point in the coordination of multiple separate expert services to provide shared decision making and timely specialist intervention.

This fine-tuned team response to acute illness has been achieved in THK ETU by a strong emphasis on education, training and quality improvement. THK ETU holds regular training for doctors and nurses within the department and across the region. These include regular bedside teaching, case based discussions, morbidity and mortality meetings and tutorials for examination preparation. THK ETU educational sessions extend regionally with regular nursing and medical workshops for external emergency staff.

\section{Scenario 4: The value of emergency medicine training}

A previously well 35-year-old female presents to THK ETU with 2 days of vomiting. The emergency doctor finds her to be febrile, pale, icteric and hypoxic with oxygen saturations of $76 \%$. History and examination are otherwise unremarkable; in particular there are no cardiac or respiratory abnormalities to explain her hypoxia. Available bedside tests are performed. A blood gas confirms profound anaemia (Haemoglobin of 6.4 $\mathrm{g} / \mathrm{dL}$,) a urine analysis suggests acute infection and ECG reveals a sinus tachycardia. The doctor formulates a problem list based on her physiology and increases oxygen delivery, places IV cannulae and commences broad-spectrum antibiotics and a blood transfusion. To further elucidate the cause of the anaemia, he performs a colour chart blot test and discovers severe methaemoglobinaemia. He withholds methylene blue as he is concerned that the patient may be G6PD deficient. Further laboratory tests are available only after management has stabilized the patient. Bilirubin is elevated $(10.1 \mathrm{mg} / \mathrm{dL})$ with otherwise normal liver function tests and a blood film is consistent with severe haemolysis. The final diagnosis of Kuppermeniya induced haemolytic crisis in the setting of G6PD is determined several days later. Despite the delay to diagnosis the patient has been safely managed. The doctor in this case has utilised a strong understanding of pathology and physiology combined with a problem based approach to the undifferentiated and acutely unwell patient. This is emphasized in the Sri Lankan Emergency Medicine MD program that he is enrolled in. 
The MD in Emergency Medicine program is integral to the successful development of emergency medicine in Sri Lanka. Emergency specialists must be experts in managing undifferentiated critically ill patients of all demographics, often with little history or collateral information. Accordingly, Emergency Medicine requires a novel training paradigm to provide experience in a broad range of specialties with an emphasis on the acute assessment and management of illness. This requires strong resuscitation, procedural and communication skills and a deep understanding of the body's physiological and pathological response to disease and treatment.

Entry into the EMMD program requires successful completion of a basic sciences examination in anatomy, physiology, pharmacology, pathology, physics and disaster medicine. The training program includes rotations through ETUs and various intensive care, anaesthetic, medical, surgical and paediatric posts where the learning emphasis is on acute care. A research task consolidates the merits of evidence-based medicine and a final fellowship examination is a test of competency set at an international standard. A year-long international rotation further provides candidates with a global perspective. The expectation is that the MD program provides Sri Lanka with experts not only in acute critical care, but practice supervision, departmental and service design and delivery of disaster medicine. There are currently 6 emergency medicine MD registrars practicing at THK ETU providing a service in direct clinical care, supervision of residents, audit, research, teaching and quality improvement.

\section{Scenario 5: The rapid relief of suffering}

A 21-year-old construction worker falls from a height of 15 feet in a workplace accident. He has multiple spinal fractures, a pelvic fracture and bilateral displaced distal tibia fracture-dislocations. His right foot is mottled, pale and pulseless. Despite $10 \mathrm{mg}$ of intravenous morphine he is screaming and writhing in pain, making spinal precautions and other assessment and management difficult. A low dose ketamine infusion is administered and the patient is soon comfortable with stable vital signs. A systematic primary and secondary survey is performed, the general surgical, orthopaedic and vascular surgeons are consulted and the patient is taken to theatre.

The ETU provides a safe environment where appropriate analgesic therapy can be safely delivered for severe pain. This case demonstrates how a strong emphasis on the early provision of adequate analgesia and sedation not only relieves suffering, but also facilitates a thorough assessment and may expedite definitive management.

\section{The future}

The THK ETU has an ongoing commitment to service improvement and a comprehensive plan for the provision of quality emergency care to the Southern Province of Sri Lanka.

There is a plan to consolidate the departmental emphasis on education and training with an expansion of the regional medical and nursing workshops and the development of a local bedside ultrasound course. The department will also offer and provide ongoing training for the EMMD candidates who will have an ongoing role in strengthening the facility.

There is also departmental support for the improvement of pre-hospital care in the area, which is currently being provided by a small number of ambulances manned by overseas trained paramedics, tuktuk drivers and helpful bystanders.

A significant impetus for the development of THK ETUs was a hope for a stronger disaster response for Sri Lanka. With ETUs at the heart of the regional disaster response THK ETU has made a strong commitment to disaster preparedness. There are designated disaster medical and nursing 'leads' within the ETU who are responsible for the authorship, distribution and resourcing of the documented disaster response plan. There are frequent hospital wide education sessions and an organised disaster stockpile cupboard at the entrance to the emergency department. There is also a vision for biannual simulated disasters in which deficiencies in the system are identified and corrected with appropriate updates in the disaster plan. One such simulation has occurred and more are planned.

There is an intention to expand a number of treatment options which have recently been introduced locally to a broader range of eligible patients. For example, emergency percutaneous coronary intervention has been offered to select STEMI patients in office hours and the ETU hopes this service may expand.

\section{Difficulties in delivering emergency care}

As Emergency Treatment Units improve, inevitable challenges arise. A particular area of focus for improvement is the timely disposition of stabilized patients. Efficient flow of patients through the ETU frees the human and physical resources for the next complex emergency and is essential to quality emergency care. Patients stabilized and appropriate for a general ward bed are transferred from the THK ETU to inpatient care with remarkable efficiency. A greater challenge is the patient requiring an ICU bed or urgent surgical management.

Difficulty in disposition of the intubated, ventilated, critically ill patients requiring intensive care is a predictable challenge that results from an increasing demand coupled with resource constraints. Those who may not have 
survived without the rapid intervention provided at the THK ETU represent an extra cohort of patients requiring an ICU bed. As in so many systems, THK ICU beds are at a premium. Currently many intubated ventilated patients have prolonged stays in the ETU. When resources allow such patients to be successfully transferred to ICU in a timely manner, they receive care in an environment and by staff more tailored to their needs, freeing ETU resources for other critically ill patients. This problem is not unique to Sri Lanka or indeed developing emergency and critical care systems, and is mirrored worldwide. In places where solutions have been found they tend to be active, complex and multifactorial; often involving engagement of hospital management, a "pull" of patients initiated from ICU, appropriate delivery of palliation and the provision of extra resources.

A second challenge of disposition is the provision of early definitive surgical interventions. With strong surgical support and the close proximity of theatre, the surgical team rapidly reviews patients requiring urgent surgery and an appropriate plan is most often made in a very timely manner. Time to surgery is dependent on theatre availability and is a focus of improvement.

\section{Conclusion}

The cases in this article have been chosen to demonstrate the current practice of emergency medicine in THK ETU. Though they typify the acuity and complexity of cases in THK ETU, they cannot convey the diversity of patients managed in the department. The process of resuscitation, stabilization and delivery to the most appropriate definitive care is performed as rapidly and as safely as possible, often in 16 acute patients in unison. On a typical day THK ETU manages 50 such patients, however in times of disaster this number may soar.

Integral to the practice of emergency medicine at THK ETU are quality triage practices, team based care, supported education and training and an emphasis on the importance of the relief of suffering. Further effort is being channeled into regional workshops, support for the EMMD program, improvements in disposition to ICU and theatre, better prehospital care and expansion of existing services.

\section{Acknowledgements}

In August 2016, a combination of profound good fortune and excellent mentorship presented my family with a four-month opportunity to live in the beautiful southern provincial city of Galle. Here I had the great privilege of working at the Teaching Hospital Karapitiya, Emergency Treatment Unit (THK ETU). I arrived in the footsteps of another Australian Emergency Physician, Dr. Nick Taylor, whose extraordinary work as a doctor, educator and mentor provided a tremendous stepping-stone for my visit. I was generously welcomed by an inspiring team of nurses and doctors, who regularly demonstrated expert care of critically unwell patients as typified by the cases in this article. My experience at THK ETU was personally and professionally hugely rewarding and it is my hope this article reflects these sentiments.

THK has been a main tertiary care provider in the South since its establishment in 1982, however at the time of the Indian Ocean tsunami of 2004, the ETU had only 2 trolleys and little formal emergency medicine training. Its remarkable expansion is a resulted of the combined efforts of strong unwavering local leads combined with remarkable international support. So much has been achieved in this department and yet there is a vision for so much more. Key parties to be acknowledged include, but are not limited to, Krishantha Jayasekera, Wimalasiri Ullawata, Pathma Gamage, Ranjana Seneviratne, Pilane Ariyananda, Priyani Senadeera, Aruna Munasinghe, Mark Fitzgerald, Gerard O'Reilly, Sue Williams, The remainder of the team at the Alfred Hospital and Royal Childrens Hospital, Melbourne, Australia, Nick Taylor and the Governments of Victoria, and Australia, all those who have called KTH ETU their workplace and so many more. We feel very privileged to have been part of this department for a short time. 